\title{
The Impact of Enhanced Recovery After Surgery on Total Joint Arthroplasty: Protocol for a Systematic Review and Meta-analysis
}

Siddharth Rele ${ }^{1,2}$, BBiomedSc; Cade Shadbolt ${ }^{1}$, BA, MA; Chris Schilling ${ }^{1}$, BEng (Hons), BCom (Hons), MSc, PhD; Nicholas F Taylor ${ }^{3,4}$, BAppSc, PhD; Michelle M Dowsey ${ }^{1,5}$, BHealthSci, MEpi, PhD; Peter F M Choong ${ }^{1,5}$, MBBS, MD

\footnotetext{
${ }^{1}$ Department of Surgery, St Vincent's Hospital, University of Melbourne, Melbourne, Australia

${ }^{2}$ Melbourne Medical School, University of Melbourne, Melbourne, Australia

${ }^{3}$ College of Science, Health and Engineering, La Trobe University, Melbourne, Australia

${ }^{4}$ Allied Health Clinical Research Office, Eastern Health, Melbourne, Australia

${ }^{5}$ Department of Orthopaedics, St Vincent's Hospital, Melbourne, Australia
}

Corresponding Author:

Siddharth Rele, BBiomedSc

Department of Surgery

St Vincent's Hospital

University of Melbourne

Level 2, Clinical Sciences Building

29 Reagent Street

Melbourne, 3065

Australia

Phone: 61390355511

Email: srele@ student.unimelb.edu.au

\section{Abstract}

Background: The number of total joint arthroplasties (TJAs) being performed is increasing worldwide. To match this increasing demand, there has been focus on hastening patients' recovery of function. This effort has culminated in the formulation of enhanced recovery after surgery (ERAS) strategies. However, with evolving ERAS programs and new recommendations, a review of current evidence is required to provide clinicians with up-to-date information about its effect on outcomes for TJA.

Objective: The objective of this study is to assess the utility of ERAS programs on patient, health service, and economic outcomes for primary, elective total hip arthroplasty (THA) and total knee arthroplasty (TKA).

Methods: A systematic search will be conducted in Medline (Ovid), EMCARE (Ovid), EMBASE (Ovid), Web of Science, CINAHL, National Health Service Economic Evaluations Database, and the Cochrane Library. Analytical, observational, and experimental designs will be included in this systematic review. Only studies including patients undergoing primary TKA and THA comparing ERAS programs with conventional surgery and postoperative care will be included. Data related to patient outcomes, health service outcomes, safety, and economic evaluation will be extracted.

Results: The search terms and primary database searches have been finalized. Findings will be reported in narrative and tabular form. Where appropriate, random effects meta-analyses will be conducted for each outcome, and heterogeneity quantified with Cochran Q test and I2 statistic. Measures of effect or mean differences will be reported with $95 \%$ confidence intervals. The results of this systematic review will be disseminated in a peer-reviewed journal.

Conclusions: This protocol will guide a systematic review assessing outcomes associated with ERAS surgery in primary THA and TKA.

Trial Registration: Open Science Framework osf.io/y4bhs; https://osf.io/y4bhs

International Registered Report Identifier (IRRID): PRR1-10.2196/25581

(JMIR Res Protoc 2021;10(3):e25581) doi: 10.2196/25581 


\section{KEYWORDS}

enhanced recovery after surgery; total knee arthroplasty; total hip arthroplasty; systematic review; meta-analysis; postoperative outcomes; economic evaluation

\section{Introduction}

The number of total joint arthroplasty (TJA) performed worldwide is increasing [1-4]. In the United States alone, the number of total hip (THA) and total knee (TKA) arthroplasties performed each year has doubled between 2000 and 2014 [5]. With this increasing demand, reducing length of stay (LOS) has become a focus as a key hospital performance indicator [6] and a method of containing procedure-level costs [7]. Decreasing LOS for TJA [8,9] has also made way for the introduction of a strategy for outpatient surgeries [10].

Despite the importance of limiting unnecessary time in hospital following TJA, it is vital that strategies that aim to decrease LOS do not come at the expense of patients experiencing inferior postoperative outcomes. Enhanced recovery after surgery (ERAS) offers one promising approach to reducing LOS by streamlining preoperative and perioperative care $[11,12]$. These principles were first promulgated by Kehlet et al [13] for colorectal surgery more than 20 years ago. More recently, growing attention has been paid to whether the principles of ERAS can be employed to reduce LOS following TJA. Despite this, prior to 2020, there were no consensus statements regarding the implementation of ERAS principles in the context of arthroplasty. Since then, the ERAS society, the body responsible for proposing recommendations for ERAS protocols in surgery, has released recommendations for perioperative care after arthroplasty $[14,15]$. However, the proposed ERAS items highlighted in these recommendations stand in contrast to those currently published in literature-namely with the use of peripheral nerve blocks and postoperative analgesia [16].

Evidence relating to the safety and efficacy of implementing ERAS pathways is fast evolving. Recent systematic reviews by Zhu et al [11] and Deng et al [12] found ERAS programs reduced both LOS and incidence of complications in the 30 days following THA and TKA, without driving a commensurate increase in re-admission following discharge. In addition, one previous review on the cost-effectiveness of ERAS pathways found ERAS surgery to be dominant compared with conventional treatment [17]. To date, available systematic reviews have yet to explore the evidence relating to other post-acute care outcomes such as emergency department visits, postoperative primary care visits, and revision surgery. Moreover, these systematic reviews have reported only total complications and re-admissions in the 30 days following surgery, and neglected to examine outcomes over a longer period or to stratify the analysis by medical and surgical complications and re-admissions $[18,19]$. Furthermore, previous cost analyses have been based on small number of studies with fewer than 50 patients in each treatment arm [20,21]. Finally, the previously published systematic reviews have not assessed the impact of the number of type of items that may be combined as part of a particular ERAS program. Rather, they have focused only on comparing cohorts undergoing surgery informed by ERAS principles and those without $[11,12,17]$.
Collectively, the limited scope of prior systematic reviews leaves us without a comprehensive picture of the potential risks associated with ERAS pathways - and with an incomplete understanding of the necessary features of a safe and effective ERAS pathway for patients undergoing arthroplasty. With these considerations in mind, the proposed systematic review and meta-analysis aims to assess the utility of ERAS programs on patient, health service, and economic outcomes for primary, elective THA, and TKA.

\section{Methods}

\section{Study Reporting and Registration}

This systematic review protocol will be reported according to the "Preferred Reporting Items for Systematic Review and Meta-analysis - Protocols" (PRISMA-P) [22] and the "Meta-Analysis of Observational Studies in Epidemiology" (MOOSE) [23] guidelines. In addition, this review has been registered prospectively with Open Science Framework. Any conflict between reviewers throughout the review process will be resolved through discussion; and if a consensus is not able to be reached, a third author will be consulted. The full search strategy for the primary databases can be found in Multimedia Appendix 1.

\section{Criteria for Inclusion}

\section{Type of Studies}

All analytical, observational, and experimental studies will be included in this systematic review. Studies that will be excluded are descriptive studies, case reports, editorials, commentaries, qualitative studies, and literature reviews. Reference lists of relevant systematic and literature reviews will be searched to find additional studies that can be included. Only studies written in English will be included.

\section{Type of Population}

The population of interest is patients undergoing primary, elective THA or TKA. Studies will also be included if patients are undergoing bilateral procedures (including simultaneous and sequential procedures), as these procedure types may determine discharge destination.

Patients undergoing revision or partial arthroplasty and those undergoing arthroplasty for fractures will be excluded. Studies involving a mixed cohort will be included provided that sufficient data are present on measures of primary, elective TJA.

\section{Type of Intervention}

For the purposes of this review, ERAS programs will include fast-track surgery. The intervention of interest is any type of ERAS recovery program, which includes more than 1 item that is distinct from the conventional treatment program. Examples of such items include use of preoperative patient education, standardized anesthetic technique, and early and intensive mobilization. 


\section{Type of Comparator}

The comparator of interest is conventional surgery and postsurgical pathway.

\section{Outcomes of Interest}

\section{Primary Outcomes}

These are centered around outcomes of arthroplasty and include patient outcomes, health service outcomes, and safety.

\section{Patient Outcomes}

Any measures of patient-reported outcome measures (eg, pain, function, global assessment, health-related quality of life) will be recorded. These will be stratified according to the timeframes examined (eg, 6 months, 1 year) up to 2 years postoperatively. In addition, measures of mobility, including, but not limited to, 6-minute walk test and walking speeding will be assessed.

\section{Health Service Outcomes}

These include LOS, discharge destination, duration of rehabilitation, primary care visits and emergency department visits, and re-admissions within 90 days of surgery. Re-admission will be categorized into medical and surgical causes, if possible [18]. Discharge destinations will be grouped into inpatient rehabilitation and discharge home; sensitivity analyses will be utilized to assess the impact of differing levels of postdischarge support, such as home with support, home without support.

\section{Safety}

This includes data on patient safety, including mortality, and any complications. All will be reported for 30 and 90 days. Complications will be grouped and organized into type of complication (eg, medical or surgical complication [18]). In addition, complications tied directly to the procedure, including surgical site infections, stiffness, manipulations under anesthesia, and revision surgery, will have no time restrictions placed.

\section{Secondary Outcomes}

These outcomes are centered around economic evaluation (any measure including, but not limited to, measures of cost-effectiveness, cost savings, total cost).

\section{Search Strategy}

To ensure that a Cochrane review has not been published on the topic of impact of reducing LOS on post-acute care, the Cochrane library has been searched using the MeSH term and keyword "Arthroplasty." This search has confirmed a Cochrane review has not been previously published on this topic.

Database searches were devised with recommendations from an external research librarian and previous literature suggesting optimal database combinations [24]. A comprehensive literature search will be performed of MEDLINE (Ovid), Web of Science, CINAHL, Emcare (Ovid), Embase (Ovid), National Health Service Economic Evaluations Database, and the Cochrane library from their inception to January 2021. In addition, Google scholar will be utilized to supplement the primary database search and reduce the risk of publication bias. Keywords and MeSH were combined with the operator "OR" for the population and intervention. The population and intervention queries were combined with the "AND" operator.

\section{Study Selection}

Following independent screening of the first $10 \%$ of studies, the agreed eligibility criteria will be refined by discussion between the 2 reviewers (SR and CSh), as per PRISMA-P guidelines [22]. This final eligibility criteria will then be utilized to independently screen titles and abstract of identified citations by 2 reviewers. Any arising discrepancies will be resolved through discussion between reviewers.

Forward and backward citation tracking of studies for full-text screening will be used to identify any additional studies. Following this, the same 2 reviewers will undertake screening of full-text studies to determine final eligibility for inclusion. A PRISMA flow diagram will be used to report the findings of this study selection process [25]. A further full-text review will be undertaken of potentially eligible studies. Interrater reliability will be assessed and will be reported as Cohen kappa statistic [26] to inform the agreement between reviewers.

Given that retrospective studies may report on the same population within the same timeframe, care will be taken to determine if these studies are independent. In the event that multiple studies are determined to be assessing the same population, all of these studies will be treated as one, and reference to all will be made, to reduce the risk of overestimation [27]. A further critical analysis of these studies will be undertaken to highlight any discrepancies, and authors will be contacted if such discrepancies are identified.

\section{Data Collection and Management}

The first round of deduplication will be undertaken through EndNote X9. After this, Covidence will be used to perform further deduplication, screening, and data extraction. STATA (version 16) software will be used to conduct statistical analyses.

Two reviewers will use a standardized data extraction form to extract data from each study independently. A further comparison of this, completed, data extraction form to assess consistency and accuracy will also be undertaken. Inconsistencies will be resolved with discussion with other authors. Study authors will be contacted via email if full-text copies of included studies are unattainable or clarification of methods or results is required.

The form for data extraction will follow items 1 to 16 in the "Reporting on ERAS Compliance, Outcomes, and Elements Research (RECOvER)" checklist [28] to inform details of the article, characteristics of patients and the details of the enhanced recovery program, and compliance within the program. The details of the employed enhanced recovery strategies will also be scored against the ERAS society's recommendation.

In addition, a results summary will be recorded with total number of patients and total number of patients with outcomes; any measure of prognostic effect (including odds ratio, relative risk and hazard ratios); standardized mean difference or mean difference and 95\% confidence intervals for each outcome measure as appropriate; use of univariate/multivariate analysis; and variables included in multivariate analysis. Patient-reported 
outcome measures will be recorded in means (SD), and any measure of economic evaluation will be recorded.

Data extraction for health economic outcomes will additionally include the following:

- Economic evaluations: economic study design, comparators, time horizon analyzed, use of discounting.

- Models: model type, model structure, assumptions, source of data (baseline and outcome of interest), model uncertainty, method of internal/external validation.

- All: costs included, source of cost data, source of utility data, quality of life instruments used, study results (currency, cost year, incremental cost effectiveness ratio).

Any missing values will be calculated provided sufficient data are available. Study authors will be contacted to obtain missing data.

\section{Risk of Bias Assessment}

Risk of bias will be assessed by 2 reviewers through the Cochrane Risk-of-Bias 2 (RoB 2) tool [29] for randomized trials and Newcastle-Ottawa Scale (NOS) [30] for nonrandomized studies. Health economic outcomes will be assessed with the Consensus on Health Economic Criteria (CHEC) list [31], and model-based studies with the questionnaire produced by the International Society for Pharmacoeconomics and Outcomes Research (ISPOR) [32]. A summary table presenting all articles included in the review will be used to present the results of the critical appraisal. Meta-analysis will be performed if articles have adequate data for quantitative synthesis, and those with a high risk of bias will be excluded in the form of a sensitivity analysis to determine their influence on the meta-analysis [33].

\section{Data Synthesis and Statistical Analysis}

All studies will be presented in both tabular and narrative form. A quantitative analysis of the findings from the included studies is planned for all outcomes. A random effects model will be utilized as heterogeneity is anticipated between studies [34]. Meta-analyses will be reported with a measure of effect (for dichotomous outcomes) and mean difference (for continuous outcomes). When different outcome measures are utilized for the same outcome, a standardized mean difference will be reported [35]. 95\% confidence intervals will be reported for all. Care will be taken to report continuous variables separately from categorical variables.

Heterogeneity will be assessed by the Cochran $Q$ test and $I^{2}$ statistic [36]. Subgroup analyses with random-effects meta-analysis will be undertaken when significant heterogeneity is encountered. If heterogeneity is not resolved through this process, exclusion of any outlying studies in the form of sensitivity analysis will be considered if a clear explanation for conflicting results is found upon review of the papers in question. Results of meta-analysis, both including and excluding papers in question, will be presented and interpreted. If variation in the results cannot be accounted for, a meta-analysis will not be conducted, and results will be presented as per the "Synthesis Without Meta-analysis" (SWiM) guidelines [37]. Pooled estimates will be reported with a forest plot and $95 \%$ confidence interval.
Several subgroup analyses are planned if data permits. Studies will be grouped according to their ERAS protocol to understand the impact of different ERAS programs on outcomes. Additionally, each ERAS protocol will be scored according to the number of ERAS society recommendations implemented to understand the cumulative impact of the ERAS recommendations $[14,15]$. The impact of varying levels of compliance within ERAS programs will also be evaluated through a subgroup analysis. A separate subgroup analysis for programs using a same-day discharge or outpatient surgery will also be conducted. Finally, the impact of patient-related factors, within ERAS surgery, on outcomes will also be explored.

Furthermore, it is also possible that a single comparator may not be determinable, as a significant portion of the included studies may present LOS as a continuous variable. As such, analyses will be undertaken with LOS as a continuous and categorical variable (categories for LOS will be formulated depending on the included studies). If significant differences in specific components of ERAS programs, such as the composition of spinal anesthesia, method of preoperative education, or duration of postoperative regional analgesia, are encountered between studies, sensitivity analyses will be used to assess the impact of these differences.

\section{Publication Bias}

Strategies employed to reduce risk of publication bias include a search strategy that does not restrict gray literature, and data from unpublished work included in meta-analysis [33,38]. Furthermore, nonreporting biases will be attempted to be identified from other factors through contour-enhanced funnel plots with an appropriate test of asymmetry for meta-analyses including at least ten studies $[38,39]$. Any potential sources of asymmetry will be explored; and a trim and fill method will be utilized to account for the possibility of publication bias if deemed appropriate [40].

In addition, outcomes mentioned in the "Methods" section of studies will be compared with those in the results during the data extraction process. In randomized trials, the final reported outcomes will be compared with a published protocol, if available. Both reviewers will assess the completed data extraction table to determine whether certain outcomes were included by most included studies but not addressed by a few. If reporting bias is found, studies will be investigated for possible explanations, and authors may be contacted to provide clarity [33].

\section{Confidence in Cumulative Evidence}

The Grades of Recommendation, Assessment, Development and Evaluation (GRADE) approach will be used by 2 reviewers, to independently assess the quality of cumulative evidence $[41,42]$. The quality of evidence will be downgraded if significant limitations are present, as evidenced from the risk of bias. In addition, evidence will be downgraded if wide confidence intervals are encountered and if statistical heterogeneity, as assessed through the $I^{2}$ statistic, is over $25 \%$ [43]. 


\section{Results}

A preliminary search of the primary databases has been conducted. The final database search was conducted in January 2021. This search will also be repeated prior to final publication of results. We plan on disseminating the findings of this systematic review in a peer-reviewed journal.

\section{Discussion}

The primary focus of previous systematic reviews published on ERAS principles have relied upon authors to propose their own definitions of ERAS surgery. With the publication of the ERAS Society's new recommendations, having objective criteria to assess these ERAS protocols allows the effects of ERAS principles to be examined with greater clarity. The results of this systematic review will help to aggregate current research and provide greater insight into the risks and benefits of reducing LOS for TJA. In addition, this review will also aid policy makers to better understand whether reductions in LOS through ERAS pathways are occurring to the detriment of the patient and health system more broadly.

\section{Authors' Contributions}

SR drafted the manuscript. SR, CSh, CSc, NT, MD, and PC contributed to the design of the review protocol. All authors approved the final manuscript.

\section{Conflicts of Interest}

None declared.

\section{Multimedia Appendix 1}

Search strategies for primary databases.

[DOCX File, 19 KB-Multimedia Appendix 1]

\section{References}

1. Culliford D, Maskell J, Judge A, Cooper C, Prieto-Alhambra D, Arden NK, COASt Study Group. Future projections of total hip and knee arthroplasty in the UK: results from the UK Clinical Practice Research Datalink. Osteoarthritis Cartilage 2015 Apr;23(4):594-600 [FREE Full text] [doi: 10.1016/j.joca.2014.12.022] [Medline: 25579802]

2. Hooper G, Lee AJ, Rothwell A, Frampton C. Current trends and projections in the utilisation rates of hip and knee replacement in New Zealand from 2001 to 2026. N Z Med J 2014 Aug 29;127(1401):82-93. [Medline: 25225759]

3. Nemes S, Gordon M, Rogmark C, Rolfson O. Projections of total hip replacement in Sweden from 2013 to 2030 . Acta Orthop 2014 Jun 23;85(3):238-243 [FREE Full text] [doi: 10.3109/17453674.2014.913224] [Medline: 24758323]

4. Inacio MCS, Graves SE, Pratt NL, Roughead EE, Nemes S. Increase in Total Joint Arthroplasty Projected from 2014 to 2046 in Australia: A Conservative Local Model With International Implications. Clin Orthop Relat Res 2017 Aug;475(8):2130-2137 [FREE Full text] [doi: 10.1007/s11999-017-5377-7] [Medline: 28488253]

5. Sloan M, Premkumar A, Sheth NP. Projected Volume of Primary Total Joint Arthroplasty in the U.S., 2014 to 2030. J Bone Joint Surg Am 2018 Sep 05;100(17):1455-1460. [doi: 10.2106/JBJS.17.01617] [Medline: 30180053]

6. National Health Performance Authority. Hospital Performance: Hospital Performance: Length of Stay in Public Hospitals 2011-12. Sydney, NSW, Australia: National Health Performance Authority; 2013.

7. Molloy IB, Martin BI, Moschetti WE, Jevsevar DS. Effects of the Length of Stay on the Cost of Total Knee and Total Hip Arthroplasty from 2002 to 2013. J Bone Joint Surg Am 2017 Mar 01;99(5):402-407 [FREE Full text] [doi: 10.2106/JBJS.16.00019] [Medline: 28244911]

8. Burn E, Edwards CJ, Murray DW, Silman A, Cooper C, Arden NK, et al. Trends and determinants of length of stay and hospital reimbursement following knee and hip replacement: evidence from linked primary care and NHS hospital records from 1997 to 2014. BMJ Open 2018 Jan 27;8(1):e019146 [FREE Full text] [doi: 10.1136/bmjopen-2017-019146] [Medline: 29374669]

9. Sarpong NO, Boddapati V, Herndon CL, Shah RP, Cooper HJ, Geller JA. Trends in Length of Stay and 30-Day Complications After Total Knee Arthroplasty: An Analysis From 2006 to 2016. J Arthroplasty 2019 Aug;34(8):1575-1580. [doi: 10.1016/j.arth.2019.04.027] [Medline: 31064724]

10. Lovald ST, Ong KL, Malkani AL, Lau EC, Schmier JK, Kurtz SM, et al. Complications, mortality, and costs for outpatient and short-stay total knee arthroplasty patients in comparison to standard-stay patients. J Arthroplasty 2014 Mar;29(3):510-515. [doi: 10.1016/j.arth.2013.07.020] [Medline: 23972298]

11. Zhu S, Qian W, Jiang C, Ye C, Chen X. Enhanced recovery after surgery for hip and knee arthroplasty: a systematic review and meta-analysis. Postgrad Med J 2017 Dec;93(1106):736-742 [FREE Full text] [doi: 10.1136/postgradmedj-2017-134991] [Medline: 28751437] 
12. Deng Q, Gu H, Peng W, Zhang Q, Huang Z, Zhang C, et al. Impact of enhanced recovery after surgery on postoperative recovery after joint arthroplasty: results from a systematic review and meta-analysis. Postgrad Med J 2018 Dec;94(1118):678-693. [doi: 10.1136/postgradmedj-2018-136166] [Medline: $\underline{\text { 30665908] }}$

13. Kehlet H. Multimodal approach to control postoperative pathophysiology and rehabilitation. Br J Anaesth 1997 May;78(5):606-617 [FREE Full text] [doi: 10.1093/bja/78.5.606] [Medline: 1 9175983]

14. Wainwright TW, Gill M, McDonald DA, Middleton RG, Reed M, Sahota O, et al. Consensus statement for perioperative care in total hip replacement and total knee replacement surgery: Enhanced Recovery After Surgery (ERAS) Society recommendations. Acta Orthop 2020 Feb 14;91(1):3-19 [FREE Full text] [doi: 10.1080/17453674.2019.1683790] [Medline: 31663402]

15. Wainwright TW. Consensus statement for perioperative care in total hip replacement and total knee replacement surgery: Enhanced Recovery After Surgery (ERAS®) Society recommendations. Acta Orthop 2020 Jun;91(3):363-319 [FREE Full text] [doi: 10.1080/17453674.2020.1724674] [Medline: $\underline{32056486}$ ]

16. Soffin EM, YaDeau JT. Enhanced recovery after surgery for primary hip and knee arthroplasty: a review of the evidence. Br J Anaesth 2016 Dec;117(suppl 3):iii62-iii72 [FREE Full text] [doi: 10.1093/bja/aew362] [Medline: 27940457]

17. Pritchard MG, Murphy J, Cheng L, Janarthanan R, Judge A, Leal J. Enhanced recovery following hip and knee arthroplasty: a systematic review of cost-effectiveness evidence. BMJ Open 2020 Jan 15;10(1):e032204 [FREE Full text] [doi: 10.1136/bmjopen-2019-032204] [Medline: 31948987]

18. Kehlet H, Jørgensen CC. Advancing Surgical Outcomes Research and Quality Improvement Within an Enhanced Recovery Program Framework. Ann Surg 2016 Aug;264(2):237-238. [doi: 10.1097/SLA.0000000000001674] [Medline: 26910201]

19. Kehlet H, Mythen M. Why is the surgical high-risk patient still at risk? Br J Anaesth 2011 Mar;106(3):289-291. [doi: 10.1093/bja/aeq408] [Medline: 21317226]

20. Brunenberg DE, van Steyn MJ, Sluimer JC, Bekebrede LL, Bulstra SK, Joore MA. Joint recovery programme versus usual care: an economic evaluation of a clinical pathway for joint replacement surgery. Med Care 2005 Oct;43(10):1018-1026. [doi: $10.1097 / 01 . m l r .0000178266 .75744 .35]$ [Medline: $\underline{16166871]}$

21. Larsen K, Hansen TB, Thomsen PB, Christiansen T, Søballe K. Cost-effectiveness of accelerated perioperative care and rehabilitation after total hip and knee arthroplasty. J Bone Joint Surg Am 2009 Apr;91(4):761-772. [doi: 10.2106/JBJS.G.01472] [Medline: 19339559]

22. Moher D, Shamseer L, Clarke M, Ghersi D, Liberati A, Petticrew M, PRISMA-P Group. Preferred reporting items for systematic review and meta-analysis protocols (PRISMA-P) 2015 statement. Syst Rev 2015 Jan 01;4:1 [FREE Full text] [doi: 10.1186/2046-4053-4-1] [Medline: 25554246]

23. Stroup DF, Berlin JA, Morton SC, Olkin I, Williamson GD, Rennie D, et al. Meta-analysis of observational studies in epidemiology: a proposal for reporting. Meta-analysis Of Observational Studies in Epidemiology (MOOSE) group. JAMA 2000 Apr 19;283(15):2008-2012. [doi: 10.1001/jama.283.15.2008] [Medline: 10789670]

24. Bramer WM, Rethlefsen ML, Kleijnen J, Franco OH. Optimal database combinations for literature searches in systematic reviews: a prospective exploratory study. Syst Rev 2017 Dec 06;6(1):245 [FREE Full text] [doi: 10.1186/s13643-017-0644-y] [Medline: 29208034]

25. Moher D, Liberati A, Tetzlaff J, Altman DG, PRISMA Group. Preferred reporting items for systematic reviews and meta-analyses: the PRISMA statement. PLoS Med 2009 Jul 21;6(7):e1000097 [FREE Full text] [doi: 10.1371/journal.pmed.1000097] [Medline: 19621072]

26. McHugh ML. Interrater reliability: the kappa statistic. Biochem Med (Zagreb) 2012;22(3):276-282 [FREE Full text] [Medline: 23092060]

27. Tacconelli E. Systematic reviews: CRD's guidance for undertaking reviews in health care. The Lancet Infectious Diseases 2010 Apr;10(4):226. [doi: 10.1016/S1473-3099(10)70065-7]

28. Elias KM, Stone AB, McGinigle K, Tankou JI, Scott MJ, Fawcett WJ, ERAS® Society and ERAS® USA. The Reporting on ERAS Compliance, Outcomes, and Elements Research (RECOvER) Checklist: A Joint Statement by the ERAS and ERAS USA Societies. World J Surg 2019 Jan;43(1):1-8 [FREE Full text] [doi: 10.1007/s00268-018-4753-0] [Medline: 30116862]

29. Higgins JPT, Altman DG, Gøtzsche PC, Jüni P, Moher D, Oxman AD, Cochrane Bias Methods Group, Cochrane Statistical Methods Group. The Cochrane Collaboration's tool for assessing risk of bias in randomised trials. BMJ 2011 Oct 18;343:d5928 [FREE Full text] [doi: 10.1136/bmj.d5928] [Medline: 22008217]

30. Wells G, Shea B, O'Connell D, Peterson J, Welch V, Losos M, et al. The Newcastle-Ottawa Scale (NOS) for assessing the quality of nonrandomised studies in meta-analyses. URL: http://www.ohri.ca/programs/clinical_epidemiology/oxford.asp [accessed 2021-02-21]

31. Evers S, Goossens M, de Vet H, van Tulder M, Ament A. Criteria list for assessment of methodological quality of economic evaluations: Consensus on Health Economic Criteria. Int J Technol Assess Health Care 2005;21(2):240-245. [Medline: 15921065]

32. Jaime Caro J, Eddy DM, Kan H, Kaltz C, Patel B, Eldessouki R, ISPOR-AMCP-NPC Modeling CER Task Forces. Questionnaire to assess relevance and credibility of modeling studies for informing health care decision making: an 
ISPOR-AMCP-NPC Good Practice Task Force report. Value Health 2014 Mar;17(2):174-182 [FREE Full text] [doi: 10.1016/j.jval.2014.01.003] [Medline: 24636375]

33. Boutron I, Page MJ, Higgins JPT, Altman DG, Lundh A, Hróbjartsson A. Chapter 7: Considering bias and conflicts of interest among the included studies. In: Higgins JPT, Thomas J, Chandler J, Cumpston M, Li T, Page MJ, et al, editors. Cochrane Handbook for Systematic Reviews of Interventions version 6.1 (updated September 2020). London: Cochrane; 2020 .

34. Borenstein M, Hedges LV, Higgins JP, Rothstein HR. A basic introduction to fixed-effect and random-effects models for meta-analysis. Res Synth Methods 2010 Apr;1(2):97-111. [doi: 10.1002/jrsm.12] [Medline: 26061376]

35. Higgins JPT, Li T, Deeks JJ. Chapter 6: Choosing effect measurescomputing estimates of effect. In: Higgins JPT, Thomas J, Chandler J, Cumpston M, Li T, Page MJ, et al, editors. Cochrane Handbook for Systematic Reviews of Interventions version 6.1 (updated September 2020). London: Cochrane; 2020.

36. Deeks JJ, Higgins JPT, Altman DG. Chapter 10: Analysing dataundertaking meta-analyses. In: Higgins JPT, Thomas J, Chandler J, Cumpston M, Li T, Page MJ, et al, editors. Cochrane Handbook of Systematic Reviews of Interventions version 6.1 (updated September 2020). London: Cochrane; 2020.

37. Campbell M, McKenzie JE, Sowden A, Katikireddi SV, Brennan SE, Ellis S, et al. Synthesis without meta-analysis (SWiM) in systematic reviews: reporting guideline. BMJ 2020 Jan 16;368:16890 [FREE Full text] [doi: 10.1136/bmj.16890] [Medline: 31948937]

38. Page MJ, Higgins JPT, Sterne JAC. Chapter 13: Assessing risk of bias due to missing results in a synthesis. In: Higgins JPT, Thomas J, Chandler J, Cumpston M, Li T, Page MJ, et al, editors. Cochrane Handbook for Systematic Reviews of Interventions version 6.1 (updated September 2020). London: Cochrane; 2020.

39. Peters JL, Sutton AJ, Jones DR, Abrams KR, Rushton L. Contour-enhanced meta-analysis funnel plots help distinguish publication bias from other causes of asymmetry. J Clin Epidemiol 2008 Oct;61(10):991-996. [doi:

10.1016/j.jclinepi.2007.11.010] [Medline: 18538991]

40. Duval S, Tweedie R. Trim and fill: A simple funnel-plot-based method of testing and adjusting for publication bias in meta-analysis. Biometrics 2000 Jun;56(2):455-463. [doi: 10.1111/j.0006-341x.2000.00455.x] [Medline: 10877304]

41. Schunemann HJ, Higgins JPT, Vist GE, Glasziou P, Akl EA, Skoetz N, et al. Chapter 14: Completing 'Summary of findings' tables and grading the certainty of the evidence. In: Higgins JPT, Thomas J, Chandler J, Cumpston M, Li T, Page MJ, et al, editors. Cochrane Handbook for Systematic Reviews of Interventions version 6.1 (updated September 2020). London: Cochrane; 2020. URL: https://training.cochrane.org/handbook

42. Rosenbaum SE, Glenton C, Nylund HK, Oxman AD. User testing and stakeholder feedback contributed to the development of understandable and useful Summary of Findings tables for Cochrane reviews. J Clin Epidemiol 2010 Jun;63(6):607-619. [doi: 10.1016/j.jclinepi.2009.12.013] [Medline: 20434023]

43. Atkins D, Best D, Briss PA, Eccles M, Falck-Ytter Y, Flottorp S, GRADE Working Group. Grading quality of evidence and strength of recommendations. BMJ 2004 Jun 19;328(7454):1490 [FREE Full text] [doi: 10.1136/bmj.328.7454.1490] [Medline: 15205295]

\author{
Abbreviations \\ CHEC: Consensus on Health Economic Criteria \\ ERAS: enhanced recovery after surgery \\ ISPOR: International Society for Pharmacoeconomics and Outcomes Research \\ LOS: length of stay \\ MOOSE: Meta-Analysis of Observational Studies in Epidemiology \\ NOS: Newcastle-Ottawa Scale \\ PRISMA-P: Preferred Reporting Items for Systematic Review and Meta-analysis - Protocols \\ RECOvER: Reporting on ERAS Compliance, Outcomes, and Elements Research \\ THA: total hip arthroplasty \\ TJA: total joint arthroplasty \\ TKA: total knee arthroplasty
}


Edited by G Eysenbach; submitted 15.11.20; peer-reviewed by T Wainwright, M Behzadifar; comments to author 12.12.20; revised version received 04.02.21; accepted 09.02.21; published 12.03.21

Please cite as:

Rele S, Shadbolt C, Schilling C, Taylor NF, Dowsey MM, Choong PFM

The Impact of Enhanced Recovery After Surgery on Total Joint Arthroplasty: Protocol for a Systematic Review and Meta-analysis JMIR Res Protoc 2021;10(3):e25581

URL: https://www.researchprotocols.org/2021/3/e25581

doi: $10.2196 / 25581$

PMID: 33709944

(CSiddharth Rele, Cade Shadbolt, Chris Schilling, Nicholas F Taylor, Michelle M Dowsey, Peter F M Choong. Originally published in JMIR Research Protocols (http://www.researchprotocols.org), 12.03.2021. This is an open-access article distributed under the terms of the Creative Commons Attribution License (https://creativecommons.org/licenses/by/4.0/), which permits unrestricted use, distribution, and reproduction in any medium, provided the original work, first published in JMIR Research Protocols, is properly cited. The complete bibliographic information, a link to the original publication on http://www.researchprotocols.org, as well as this copyright and license information must be included. 